\title{
SITE-SELECTIVE SPECTROSCOPY AND LEVEL ORDERING IN C-PHYCOCYANINE
}

\author{
W. KÖHLER, J. FRIEDRICH \\ Physikalisches Institut und BIMF, Universität Bayreuth, Postfach 101251, D-8580 Bayreuth, Federal Republic of Germany \\ R. FISCHER and H. SCHEER \\ Botanisches Institut, Universität München, D-8000 Munich, Federal Republic of Germany
}

Received 22 September 1987

\begin{abstract}
We present a combined fluorescence and hole-burning study of the biliprotein C-phycocyanin. Sharp zero-phonon holes compare with a broad structureless fluorescence. This finding is rationalized in terms of the special level structure in this pigment, the fast energy-transfer processes and a lack of correlation of the energies of the emissive states.
\end{abstract}

\section{Introduction}

Antenna pigments are biopolymers which contain several dye molecules which act as light absorber and energy transmitter (for reviews, see refs. [1,2]). Among these antennas, the biliproteins are probably the best characterized systems to date. They contain bile-pigment chromophores which are covalently bound to the apoproteins. The conformation of the polymer chain [3] and the chromophore structures [1] produce certain sites which in turn result in a well defined level ordering of the various $S_{1}$ states of the chromophores [4]. Due to the large energy gaps and the generally weak coupling among the chromophores, the phycobiliproteins have been characterized in detail in recent years. They are antenna pigments of blue-green, red and cryptophyte algae, and are present in the former two as large, supramolecular aggregates, e.g. the phycobilisomes [1]. The results obtained on these pigments led investigators to classify the dye molecules as s (sentizing) and $f$ (fluorescing) chromophores [5,6] (for a refined treatment, see ref. [7]). Naturally, the f chromophore is the dye molecule with the lowest $S_{1}$ energy. Clearly, the level ordering within the visible band of the pigments is important with respect to the photophysics and function of antenna pigments [8]. Among the numerous papers on the photophysics of biliproteins, there are a few studies which are based on the spectral hole-burning technique [9-11] (for a review, see ref, [12]), At very low temperatures narrow dips (holes) could be burnt into the absorption of the pigments by irradiating the sample with narrow-bandwidth laser light. The detailed nature of the hole-burning photoreaction is, as yet, unknown. These studies revealed that the optical transition of the pigments are characterized by sharp zero-phonon lines. The occurrence of zero-phonon lines indicates that the excitation process of the chromophore does not lead to a reorganization of the "lattice" structure. Usually, zero-phonon lines are related to a rather rigid "lattice". It is interesting to note that recent hole-burning studies on photosynthetic reaction centers of Rhodopseudomonas sphaeroides and Rhodopseudomonas viridis [13,14] revealed extremely broad holes which originate probably from a strong electron-phonon coupling in these systems [15].

In the case of the biliproteins where the absorption transitions are characterized by sharp zero-phonon holes, one would expect that the fluorescence would also show a zero-phonon line structure, since absorption and emission are connected by a symmetry relation. However, this is not the case. The fluorescence is essentially broad and structureless, irrespective of the mode of excitation. In this paper we address this problem by comparing hole-burning and fluorescence spectra. We show that this puzzle is a consequence of the special level ordering in the pigment and the concomittant lack of correlation. 


\section{Experimental}

The sample investigated is C-phycocyanine (PC) from Mastigocladus laminosus [16] dissolved in ethylene glycol/water $(3: 1, \mathrm{v} / \mathrm{v})$ at a concentration of approximately $10^{-5} \mathrm{~m}$. At this level of concentration PC aggregates to trimers. Excitation of the sample was performed with a Coherent-599 ion-dye laser system, with a bandwidth of $10 \mathrm{MHz}$. For hole burning typical power levels were $20 \mu \mathrm{W}$ and burning times were of the order of $100 \mathrm{~s}$. The holes were detected by observing the fluorescence while scanning the laser in absorption. The resolution in this excitation spectroscopy is determined by the bandwidth of the laser $\omega_{\mathrm{L}}(10 \mathrm{MHz})$. The fluorescence spectra were measured with a $1 \mathrm{~m}$ monochromator whose resolution was set to about $1 \mathrm{~cm}^{-1}$.

The absorption and hole-burning experiments were carried out at $4.2 \mathrm{~K}$. Fluorescence measurements were performed at $25 \mathrm{~K}$. At this temperature the Debye-Waller factor is still high enough to observe zero-phonon emission in case there is any. On the other hand, photobleaching is much less at elevated temperatures, due to a larger homogeneous linewidth and, hence, if there were a zero-phonon line, it is much easier to detect.

\section{Results}

Fig. 1 shows the absorption spectrum of PC together with a hole burnt at the position indicated by the arrow. This narrow hole $\left(0.12 \mathrm{~cm}^{-1}\right.$ at $\left.4.2 \mathrm{~K}\right)$ demonstrates the zero-phonon character of the transition.

Fig. 2 summarizes the results of a whole series of experiments. Shown is a series of fluorescence spectra excited at various positions within the absorption spectrum. Several points are worth stressing: First, there is no quasi-line structure in the fluorescence. Second, though there are some subtle changes in the long-wavelength wing of the spectrum (see below), the peak position and the width of the band do not seem to depend on the excitation wavelength. As a consequence, there is a very uncommon but characteristic feature of the fluorescence spectrum of the pigment: Exciting the red edge of the band the gradual onset of the fluorescence disappears completely.

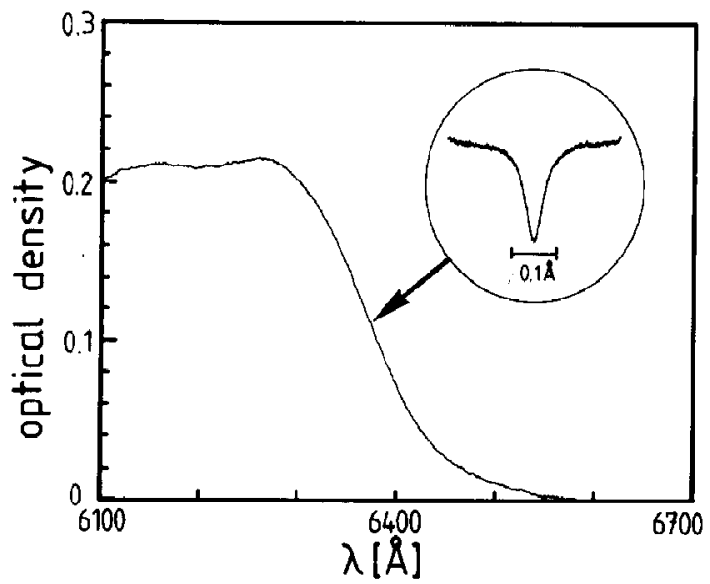

Fig. 1. Absorption spectrum of $C$-phycocyanin. The insert shows a zero-phonon hole burnt at the wavelength indicated by the arrow. Temperature $4.2 \mathrm{~K}$.

Instead there is a sharp cutoff to the blue of the laser frequency. This phenomenon is most obvious in fig. 2c. Further, the relative intensity in the long-wavelength tail decreases and the main features of the line seem to narrow a little as the laser is tuned to the red.

\section{Discussion}

The above findings, i.e. the discrepancy between the sharp zero-phonon structure in absorption and the broad featureless fluorescence can be rationalized by the special level ordering in the pigment and the lack of correlation. C-phycocyanine contains three types of chromophores $[3,4,17]$. The excited states of the chromophores feel the disorder in the microscopic environment, and, consequently, are inhomogeneously broadened. At the moment we cannot definitely say whether the disorder stems from a disordered protein or from the glass-forming solvent molecules or from both. To explain the puzzle of a sharp structured absorption and a featureless fluorescence we make three assumptions which fit well into the background of experimental data on biliproteins:

Assumption 1 is that there is no single well defined emissive state, but at least two of them (which we call $C_{1}$ and $C_{2}$ in the following). Should the inhomogeneous distributions of the two states cover ex- 


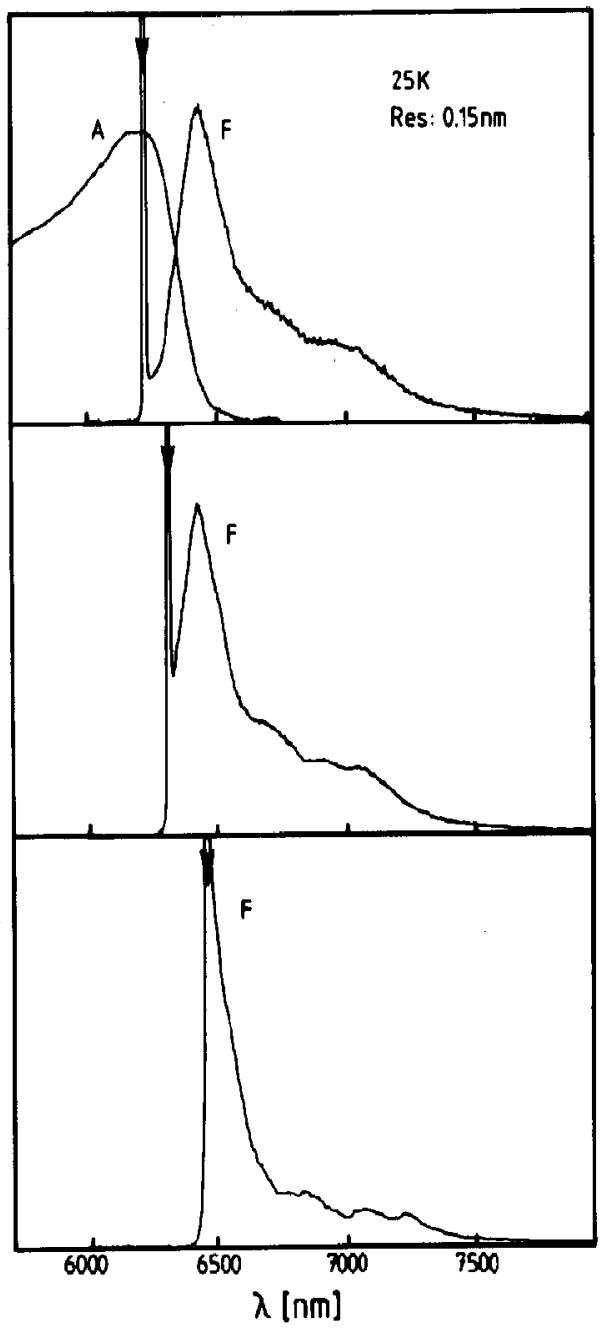

Fig. 2. Fluorescence spectrum of $\mathrm{C}$-phycocyanin as a function of excitation wavelength indicated by the arrow. Note that the band maximum does not shift and that no obvious line narrowing occurs.

actly the same spectral range, it is then impossible to excite one of them separately. For the problem considered here it is sufficient to assume that the two distributions coincide within their small energy range.

Assumption 2 is that these two ${ }^{\# 1}$ states are coupled by an effective energy transfer.

Whereas assumptions 1 and 2 are based on a great deal of experimental data, assumption 3 is novel in

\footnotetext{
\#1 More than two degenerate states would not change the
} argumentation.

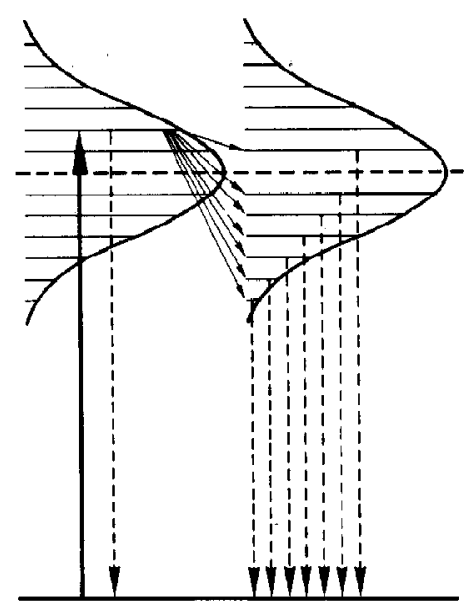

Fig. 3. Strongly overlapping emissive bands without energy correlation and coupled by fast transfer proccsscs can account for the observed features of sharp zero-phonon absorption lines and unstructured emission.

this context: We assume that there exists no correlation between the energies of two emissive states $C_{1}$ and $C_{2}$. That is, for an individual molecule any splitting (see fig. 4 below) within the spectral bandwidth is possible. The situation is characterized in fig. 3 : The excitation selects a certain energy level within the inhomogeneous band. Since there is no energy correlation on the one hand and an effective energy transfer on the other hand, all those sites with energies smaller than $\omega_{L}$ are occupied according to their density of states, resulting in a featureless fluorescence. The energy range above the excitation frequency can, of course, not be populated at low temperatures and, hence, the fluorescence band shows a cutoff at the laser frequency. It is worthwhile stressing that the same conclusion, namely an emissive state degenerate within its inhomogeneous contours, was reached for phycoerythrin [8]. It was, however, based on totally different experiments, namely fluorescence studies of the isolated subunits of the pigment.

The lack of correlation indicates that either the protein itself is disordered in solution or that the random interaction of the chromophores with the amorphous glass is much stronger than the chromophore-protein interaction, so that solvent-induced disorder prevails. The latter situation could 
easily occur in case the chromophores are on the surface of the protein and/or are strongly exposed to the random fields of the glass matrix.

There is biochemical evidence that all chromophores are accessible to the solvent and that the chromophore $\beta 84$ can even be reached by larger molecules $[3,4]$. There are also indications that at least one of the chromophores, e.g. $\beta$ 84, which happens to be the "f chromophore", is somewhat mobile within the protein $[3,4]$.

An interesting observation in this context is the fact that the hole-burning efficiency increases strongly to the red of the visible band. For example, the light fluxes needed to burn holes of the same relative depth differ by as much as a factor of 10 between the red edge of the band and the band maximum.

This observation cannot solely be accounted for by the increasing probability of phonon excitation [18]. Instead, we interpret this phenomenon by assuming that only the fluorescing chromophore in this protein is effectively burnt, whereas all sensitizing chromophores are much less affected by the photochemistry due to fast energy transfer to the fluorescing chromophore. The $s$ to $f$ transfer times are one to two orders of magnitude faster than the fluorescence decay time $[19,20]$. Clearly, considering the inhomogeneously broadened spectra the concept of $s$ and $f$ chromophores breaks down because, due to the strong spectral overlap, the fluorescing state is not well defined. $C_{2}$ as well as $C_{1}$ are sensitizing and fluorescing. However, in an isolated molecule, there are, indeed, well defined $s$ and $f$ states. In fig. 4 we depicted two situations. In the case shown in fig. $4 a$ the excited $C_{1}$ state is higher in energy than $C_{2}$. Hence, $C_{1}$ is sensitizing and $C_{2}$ is fluorescing. Such a molecule is unlikely to be burnt. In case (b) $C_{1}$ is lower in energy than $C_{2}$, and hence is the fluorescing state. In this case hole burning can occur or resonance fluorescence may be emitted. It is immediately obvious that, as the exciting laser frequency is tuned to the red edge of the band, the probability of finding a $\mathrm{C}_{2}$ state with lower energy at the same molecule is highly diminished and hence the probability of burning is increased. Since the hole-burning reaction yield is not unity, it is clear that the probability of resonance emission increases simultaneously. Hence, there is an onset of structured emission as can be seen in the change of the vibronic tail of the

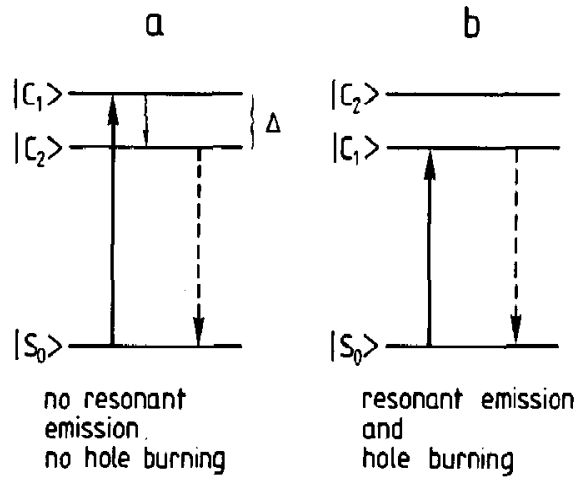

Fig. 4. The uncorrelated emissive states as shown for two individual biliprotein molecules. Resonance fluorescence and hole burning occur only if, in an individual molecule, the lowest state is occupied (b). Due to the lack of correlation the states $C_{1}$ and $\mathrm{C}_{2}$ can be anywhere in the inhomogeneous band and hence can exchange their fluorescing and sensitizing properties.

fluorescence which loses intensity and sharpens its structure. Also the vibrational pattern starts to shift with the excitation frequency as the laser is tuned into the red edge. We note that correlation within the energy-transfer states was a point in explaining the hole pattern in phycoerythrin [10]. It seems worthwhile to investigate this aspect again by doing fluorescence line-narrowing experiments.

\section{Summary}

In this paper we explain the long-known discrepancy between the sharp zero-phonon line structure in absorption and the rather featureless emission. This phenomenon is based on the occurrence of several special features.

First, there is not a well defined lowest excited state. Instead there are at least two of them which cover the same spectral range with their inhomogeneously broadened band. Second, all states are coupled via fast energy-transfer processes. Third, there is no correlation between the two lowest states. As a consequence, for example, if one populates a state $C_{1}$ in a certain molecule the corresponding state $\mathrm{C}_{2}$ may be found anywhere within the inhomogeneously broadened band. Excitation at the red edge induces some amount of energy selectivity in the fluores- 
cence spectrum, since the probability of finding an acceptor state with a lower energy is drastically reduced. It seems that the conclusions made do not depend on whether the pigment is in a monomeric or a trimeric state, as long as the transfer time in the monomer is short enough. This, however, seems to be the case [21]. We finally note that the situation may be different in other solvents. For example, in a frozen solution of saccharose/phosphate buffer, we found a weak onset of quasi-line structure in emission and a richer structure in absorption with much narrower inhomogeneous lines.

\section{Acknowledgement}

The authors acknowledge many discussions with A. Blumen and D. Haarer. Financial support was given by the Deutsche Forschungsgemeinschaft, SFB 213 and Forschergruppe "Pflanzliche Tetrapyrrole". HS acknowledges the mass culture of Mastigocladus laminosus by Dr. Reng at the Gesellschaft für Biotechnologie, D-3301 Stöckheim.

\section{References}

[1] H. Scheer, Angew. Chemie 93 (1981) 230; Angew. Chem. Intern. Ed. Engl. 20 (1981) 241.

[2] R.J. Cogdell and H. Scheer, Photochem. Photobiol. 42 (1985) 669.

[3] T. Schirmer, W. Bode, R. Huber, W. Sidler and H. Zuber, J. Mol. Biol. 184 (1985) 257.
[4] S. Siebzehnrübl, R. Fischer and H. Scheer, Z. Naturforsch. 42C (1987) 258.

[5] R.E. Dale and F.W.J. Teale, Photochem. Photobiol. 12 (1970) 99.

[6] F.W.J. Teale and R.E. Dale, Biochem. J. 116 (1970) 161.

[7] K. Sauer and H. Scheer, Photochem. Photobiol., submitted for publication.

[8] B. Zickendraht-Wendelstadt, J. Friedrich and W. Rüdiger, Photochem. Photobiol. 31 (1979) 367.

[9] J. Friedrich, H. Scheer, B. Zickendraht-Wendelstadt and D. Haarer, J. Am. Chem. Soc. 103 (1981) 1030.

[10] J. Friedrich, H. Scheer, B. Zickendraht-Wendelstadt and D. Haarer, J. Chem. Phys. 74 (1980) 2260.

[11] J. Friedrich, H. Scheer, B. Zickendraht-Wendelstadt and D. Haarer, J. Luminescence 24/25 (1981) 815.

[12] J. Friedrich and D. Haarer, Angew, Chemie 96 (1984) 96; Angew. Chem. Intern. Ed. Engl. 23 (1984) 113.

[13] S.R. Meech, A.J. Hoff and D.A. Wiersma, Chem. Phys. Letters 121 (1985) 287.

[14] S.G. Boxer, D.J. Lockart and T.R. Middendorf, Chem. Phys. Letters 123 (1986) 476; FEBS 200 (1986) 237.

[15] J.K. Gillie, B.L. Fearey, J.M. Hayes, G.J. Small and J.H. Golbeck, Chem. Phys. letters 134 (1986) 316.

[16] W. John, R. Fischer, S. Siebzehnrübl and H. Scheer, in: Antennas and reaction centers of photosynthetic bacteria, $\mathrm{ed}$. M.E. Michel-Beyerle (Springer, Berlin, 1985) p. 17.

[17] M. Mimuro, P. Flüglistaller, R. Rümbeli and $H$. Zuber, Biochim. Biophys. Acta 848 (1986) 155.

[18] A. Elschner and H. Bässler, Chem. Phys. 112 (1987) 285.

[19] A. Holzwarth, Photochem. Photobiol. 43 (1986) 707.

[20] S. Schneider, P. Geiselhart, T. Mindl, F. Dörr, W. John, R. Fischer and H. Scheer, in: Antennas and reaction centers of photosynthetic bacteria, ed. M.E. Michel-Beyerle (Springer, Berlin, 1985) p. 26.

[21] T. Gilbro, unpublished results. 